\title{
Excelência profissional: a convergência necessária de variáveis psicológicas
}

\author{
Professional excellence: The necessary convergence \\ of psychological variables
}

\author{
Leandro da Silva ALMEIDA ${ }^{1}$ \\ Solange Muglia WECHSLER ${ }^{2}$
}

\begin{abstract}
Resumo
O estudo da excelência humana assume essencialmente dois enfoques, um deles acentuando as variáveis psicológicas dos indivíduos e outro enfatizando as aquisições decorrentes da prática ou treino deliberado. Neste artigo procurou-se integrar tais estudos, propondo que o surgimento e a manutenção da excelência pressupõem uma constelação de variáveis psicológicas que vão para além da alta capacidade intelectual. Mais concretamente, descreveu-se um conjunto de variáveis cognitivas, motivacionais e de personalidade, assumidas como relevantes na excelência profissional em diferentes áreas de desempenho e de conhecimento. No seio das variáveis cognitivas e, fazendo uma ponte com as variáveis de personalidade, destaca-se o papel da criatividade para a excelência, reconhecendo a sua particular relevância em algumas áreas de realização profissional.
\end{abstract}

Palavras-chave: Cognição; Criatividade; Excelência; Motivação; Personalidade.

\begin{abstract}
The study of human excellence can be described based on two main approaches focusing either on individuals' psychological variables or on acquisitions by training or deliberate practice. The purpose of this study is to integrate these models based on the assumption that the emergence and maintenance of excellence requires a constellation of fundamental psychological variables that transcend intellectual capacity. More specifically, a set of cognitive, motivational, and personality variables that are considered important for professional excellence in different levels of performance and areas of knowledge were described. Among the cognitive variables and their association with personality variables, it is worth mentioning the significant role of creativity in the pursuit of excellence, recognizing its relevance to specific areas of professional performance.
\end{abstract}

Keywords: Cognition; Creativity; Excellence; Motivation; Personality.

As altas habilidades e talentos do ser humano sempre despertaram alguma curiosidade, mesmo que nem sempre foram entendidas ou valorizadas como um aspecto positivo. Um livro muito bem

\footnotetext{
$\nabla \nabla v$

${ }^{1}$ Universidade do Minho, Instituto de Educação. Campus de Gualtar, 4715, Braga, Portugal. Correspondência para/Correspondence to: L.S. ALMEIDA.E-mail: <leandro@ie.uminho.pt>.

2 Pontifícia Universidade Católica de Campinas, Centro de Ciências da Vida, Programa de Pós-Graduação em Psicologia. Campinas, SP, Brasil.
} 
escrito, um recorde mundial ultrapassado numa prova de atletismo, o brilhantismo na execução de um instrumento musical ou uma pesquisa que desvenda um mistério da ciência chamam a nossa atenção pela sua singularidade. No entanto, a Psicologia, desde as suas origens, voltou-se preferencialmente para a compreensão dos transtornos psíquicos, para as doenças mentais e as deficiências cognitivas.

Recentemente, em boa medida com o desenvolvimento da Psicologia Positiva, novos constructos passaram a ser pesquisados, entre eles o otimismo, a coragem, o perdão, a perseverança, a virtude, a sabedoria, o bem-estar, a sobredotação e a excelência (Seligman \& Csikszentmihalyi, 2000). Embora a Psicologia Humanista já viesse discutindo a importância de se acreditar no potencial humano (Maslow, 1968; Rogers, 1961), a intervenção de Martin Seligman, na qualidade de presidente da American Psychology Association, pode ser apontada como decisiva para a emergência ou a consolidação da Psicologia Positiva. A saúde, a realização excelente ou o bem-estar são temas tão meritórios do estudo da Psicologia quanto as doenças, as disfunções e o sofrimento psíquico (Linley, Joseph, Harrington, \& Wood, 2006).

A Psicologia Positiva, segundo Seligman (2002), valoriza as experiências de bem-estar, satisfação e otimismo face ao futuro, centrando-se nos processos que explicam o desenvolvimento e o funcionamento ótimo do indivíduo, dos grupos e das instituições (Gable \& Haidt, 2005; Sheldon \& King, 2001). Sendo verdade que experiências e sentimentos negativos podem também estimular o desenvolvimento psicológico do indivíduo (McNulty \& Fincham, 2012), a Psicologia Positiva centra-se nos constructos e nos processos que podem favorecer a felicidade e a qualidade de vida, o bem-estar subjetivo e realização plena (Seligman, 2002). Transpondo para a excelência, a investigação procura identificar os fatores e os processos que explicam a emergência e a manutenção de um desempenho superior na área acadêmica e profissional (Ericsson, 2005; Monteiro, Castro, Almeida, \& Cruz, 2009; Renzulli, 2002; Zimmerman, 2002).

Tendo como foco deste artigo a excelência

768 profissional, procurou-se sistematizar a investigação psicológica que busca explicar a expertise de indivíduos adultos no trabalho, nos esportes, nas artes, na vida social ou na investigação científica (Lubinski \& Benbow, 2000; Shanteau, Weiss, Thomas, \& Pounds, 2002; Winner, 2000). Ao mesmo tempo, a presente investigação se deu nos domínios da inteligência e da criatividade (Almeida, Guisande, \& Ferreira, 2009; Wechsler, 2006; Wechsler, Oliveira, \& Suárez, 2015), bem como de estudos nos campos da sobredotação e excelência (Araújo, Cruz, \& Almeida, 2011; Garcia-Santos \& Almeida, 2008; Garcia-Santos, Almeida, \& Werlang, 2012; Matos, Cruz, \& Almeida, 2011; Monteiro, Almeida, \& Vasconcelos, 2012; Peiffer \& Wechsler, 2013). Trata-se de uma síntese reflexiva na área, sem especificar ou diferenciar domínios profissionais de atuação, que pretende destacar a relevância das variáveis psicológicas na emergência e manutenção da excelência e, assim, sugerir caminhos para a investigação psicológica no estudo de pessoas excelentes ou para o apoio psicológico à sua realização profissional.

\section{Conceito e explicação da excelência}

De forma bem simples, entende-se a excelência, neste artigo, como realização particularmente singular ou desempenho notoriamente superior numa determinada área de atuação ou domínio de conhecimento (Trost, 2000). Aspectos quali e quantitativos combinam-se na definição desse conceito, podendo uns e outros tornarem-se mais e menos importantes dependendo da área de excelência profissional investigada (Araújo et al., 2011; Garcia-Santos \& Almeida, 2008). Um primeiro problema passa, então, pela especificação dos critérios da excelência profissional, mesmo quando se institui que o conceito se refere aos indivíduos pertencentes aos 5\% com desempenho mais elevado (critério estatístico). Se em várias modalidades esportivas existem rankings internacionais que podem servir esse objetivo, também podendo-se recorrer às medalhas conquistadas em campeonatos do mundo ou nos Jogos Olímpicos, nas outras áreas a situação tende a não ser tão clara e os critérios de definição da excelência assumem alguma ambiguidade. Por exemplo, se nas artes, música e litera- 
tura pode ser determinante a marca ou o cunho identitário do autor, na investigação científica pode-se tomar alguns critérios quantitativos, tais como verbas auferidas para financiamento dos projetos ou quantidade de artigos publicados em revistas internacionais.

A investigação psicológica na área da excelência está marcada por dois enfoques: um primeiro enfatiza, sobretudo, as variáveis psicológicas e o seu desenvolvimento, ou seja, os atributos dos indivíduos (Gagné, 2004; 2015; Renzulli, 2002; Renzulli \& Gaesser, 2015), enquanto um segundo associa a excelência aos conhecimentos e competências decorrentes de uma extensa experiência ou prática deliberada na área (Ericsson, 2005; Lubinski, Benbow, Shea, Eftekhari-Sanjaui, \& Halvorson, 2001; Lubinski, Benbow, Webb, \& Bleske-Rechek, 2006). Neste artigo, mesmo reconhecendo a relevância do contexto cultural e da prática deliberada, pretende-se destacar as variáveis psicológicas que explicam a emergência e a manutenção da excelência profissional em indivíduos adultos. Acredita-se, aliás, que a própria prática deliberada apenas se institui e surte efeito quando certas variáveis psicológicas dos indivíduos estão presentes (Matos et al., 2011). Assim, será destacada uma constelação de variáveis cognitivas, motivacionais e de personalidade para a explicação da excelência.

\section{Variáveis psicológicas na explicação da excelência}

A excelência deve ser entendida como um fenômeno multivariado, podendo algumas variáveis ganharem relevância sobre outras em função das áreas de realização. Basicamente, podemos assumir que as habilidades cognitivas, mesmo que necessárias, não são suficientes para explicar a excelência, menos ainda quando esta está relacionada a algumas áreas da realização artística e esportiva, por exemplo.

Num dos modelos mais referenciados internacionalmente, Renzulli (1986; 2002) postula que a sobredotação traduz a interseção de três variáveis (daí chamar-se a "teoria dos três anéis"): aptidão intelectual acima da média, criatividade e motivação ou envolvimento na tarefa. Essa teoria tem sofrido algumas modificações, aceitando-se a possibilidade de indivíduos sobredotados serem mais orientados por uma inteligência convergente/acadêmica (o tradicional QI - Quociente de Inteli-gência) ou pelas habilidades criativas (Renzulli \& Gaesser, 2015), discutindo-se ainda hoje a relativa independência entre esses dois aspectos, sobretudo quando se considera sujeitos muito inteligentes ou muito criativos. Progressivamente, alguns autores (Gagné, 2004; 2015; Winner, 2000) postulam as habilidades cognitivas na base dos talentos e de desempenhos superiores, destacando um processo de desenvolvimento em que as capacidades básicas indiferenciadas se cristalizam em aptidões específicas, fruto das experiências e das motivações dos indivíduos (Ackerman, 1996; Gagné, 2015). Nesse quadro, é clara a relevância das variáveis psicológicas e das próprias capacidades básicas, pois que, sem elas, o treino ou a aprendizagem não moldam talentos.

Nessa mesma linha, o Modelo do Desenvolvimento da Expertise de Sternberg (1998; 2001) propõe que as altas habilidades decorrem de um processo de aquisição e cristalização de um conjunto de capacidades consideradas necessárias para uma elevada performance, seja em uma ou várias áreas de desempenho. Depreende-se, então, a importância de destacar as variáveis cognitivas, motivacionais, criativas e de personalidade que podem explicar a excelência humana. A criatividade merece atenção particular, pois que, como fenômeno multidimensional, integra ela própria variáveis cognitivas, de personalidade e sociais (Sainz et al., 2011; Wechsler, 2008).

\section{Variáveis cognitivas da excelência}

Ainda que não sendo suficiente, a cognição é variável necessária da excelência. Mesmo quando se fala sobre talento motor ou excelência em áreas do desporto e atividade física as habilidades cognitivas são requeridas. No entanto, importa entender essas variáveis cognitivas muito para além da capacidade intelectual (QI), incluindo então o conhe- 
cimento e os processos metacognitivos e autorregulatórios, a título de exemplo. Na realidade, no estudo da excelência deve-se ampliar o próprio conceito de cognição não o restringindo ao QI ou às capacidades abstratas de pensar e raciocinar, numa referência ao fator $g$ ou inteligência fluída (Almeida et al., 2009; Primi, 2002).

Assim, a investigação aponta que os indivíduos excelentes apresentam na sua área uma quantidade de conhecimento superior, para além de um saber mais profundo e mais bem organizado, sugerindo, assim, que a prática deliberada pode favorecer a aquisição, o armazenamento organizado e a evocação de tal conhecimento. Num estudo clássico com jogadores de xadrez, Chase e Simon (1973) constataram desempenhos superiores por parte daqueles que conseguiam ter disponível uma maior quantidade de informações (em termos de posições de jogadas) na sua memória a longo prazo, estando esse número de jogadas memorizadas associado aos anos de prática. Decorre daqui, aliás, a "regra dos dez anos de prática" necessários para atingir a excelência em diferentes domínios de realização (Chase \& Simon, 1973; Ericsson, Krampe, \& Tesch-Homer, 1993).

Por outro lado, indivíduos com excelência numa dada área apresentam comportamentos metacognitivos e de autorregulação da sua realização que os diferenciam claramente. Para Sternberg (1998) existe um conjunto de capacidades metacognitivas que possibilitam o desenvolvimento da excelência, como por exemplo o desenvolvimento de esquemas apurados de classificação dos problemas, a automatização de sequências na resolução dos mesmos e a monitorização do implemento das ações e avaliação das soluções. Por sua vez, Zimmerman (2002) descreve um conjunto de processos autorregulatórios, como planificação, monitorização e avaliação, associados ao desempenho excelente. Vale acrescentar que esses processos estimulam sentimentos ajustados de autoeficácia e de autoestima, os quais reforçam a energia e paixão pela aprendizagem e realização (Ericsson \& Lehman, 1996).

No quadro das variáveis cognitivas as770 sociadas à excelência, a criatividade assume lugar de destaque. Para alguns autores a criatividade e a inteligência são constructos independentes (Getzels \& Jackson, 1962; Runco, 2007), embora outros defendam uma relação positiva entre ambos, assumindo, por exemplo, que indivíduos os quais atingem altos níveis de conceituação e abstração também geram novas ideias (Nusbaum \& Silvia, 2011; Sternberg, 2001). Aliás, a teoria do limiar (threshold) afirma que os dois constructos se tornam mais autônomos entre si a partir de um ponto de corte, como um QI de 120, complementando-se então sua especificidade na explicação da excelência (Jauk, Benedek, Dunst, \& Neubauer, 2013; Lubart, 2007). Nesse sentido, e mesmo assumindo que a criatividade é uma variável psicológica que vai para além da inteligência (Sainz et al., 2011; Wechsler, 2008), ambas convergem para a excelência na idade adulta (Runco, Millar, Acar, \& Crammond, 2010), justificando a sua estimulação na infância e adolescência (Pfeiffer \& Wechsler, 2013; Wechsler, 2006; 2008) e o seu maior estudo em contextos profissionais (Nakano, 2007).

\section{Variáveis motivacionais da excelência}

No quadro dos fatores pessoais associados à excelência profissional, alguns autores destacam variáveis motivacionais, como determinação e comprometimento com as tarefas (Ryan \& Deci, 2004; Stoeber \& Otto, 2006). Sem investimento pessoal e determinação do indivíduo em fazer bem ou em melhorar sucessivamente o seu desempenho em função do treino ou da prática, não parece ser possível atingir a excelência. Com efeito, as biografias de pessoas excelentes nos mais diversos domínios destacam a sua persistência e paixão por aprender e executar bem (Araújo et al., 2011; Vallerand, 2008). Esses níveis elevados de motivação intrínseca confundem-se a partir da adolescência com necessidades de autonomia, competência e afiliação (Vallerand, 2008; 2010), emergindo então uma paixão ou dedicação extrema às aprendizagens e realização. Nestes casos, ao invés de uma paixão patológica decorrente de pressões externas introjetadas, fala-se de um sentimento harmônico inerente ao self (Deci \& Ryan, 2000; Ryan \& Deci, 2004). 
Apenas a paixão por determinada atividade pode explicar a perseverança e o elevado envolvimento pessoal na melhoria constante da sua execução (Stoeber \& Otto, 2006; Vallerand, 2008). Na realidade, só esse sentimento pode explicar o entusiasmo e a dedicação ao aperfeiçoamento diário constante, as várias horas de treino ao longo de meses e anos para atingir e manter a excelência (Deci \& Ryan, 2000; Ryan \& Deci, 2004; Vallerand, 2008). A paixão traduz-se num estado de intenso envolvimento (engagement) e absorção na tarefa, denominado por Csikszentmihalyi (1997) como "fluir" (flow). Nessa situação, o indivíduo perde a noção das horas, vivenciando o imenso prazer que a atividade the produz, estando esse sentimento diretamente relacionado à busca da realização pessoal e profissional. Quando essas pessoas são entrevistadas, podemos notar no seu discurso algumas evidências da paixão que nutrem por uma dada atividade: (i) procuram realizar tais atividades todo o tempo dedicando quantidades elevadas de energia; (ii) atingem níveis altos de envolvimento na atividade e experienciam o crescimento pessoal; (iii) obtêm índices elevados de prazer e bem-estar na atividade; e (iv) sentem que a sua identidade passa pelo fato de realizar bem essa atividade (Araújo et al., 2011; Vallerand, 2008; 2010).

A paixão exemplifica a relevância da motivação intrínseca na obtenção e manutenção de altos níveis de excelência (Amabile \& Mueller, 2008). Essa motivação, por sua vez, responde por necessidades pessoais inerentes à própria aprendizagem e realização. Com frequência os profissionais de excelência tornam-se perfeccionistas, assumindo internamente o seu sucesso e impondo a si próprios exigências de qualidade crescente nas suas tarefas profissionais (Frost, Marten, Lahart, \& Rosenblate, 1990; Stoeber \& Otto, 2006). É comum refazerem o mesmo trabalho muitas vezes, investindo uma grande quantidade de tempo e energia para alcançar o nível elevado de qualidade, o que, por vezes, os torna demasiado autocríticos e os leva a ter medo do fracasso (Alencar, 2007). Sendo uma característica positiva e moderadora na excelência, importa também estarmos atentos a formas mais desestruturantes da personalidade (perfeccionismo obsessivo- -compulsivo), quando a mesma se traduz na fixação de padrões de realização irrealistas fragiliza a autoestima, institui medo crônico do fracasso ou gera sentimentos de vergonha, insegurança e ansiedade desmesurada. Essa situação, por exemplo, é contrária a uma paixão harmônica pelo trabalho pautada na abertura à experiência e tolerância à ambiguidade em profissionais criativos (Runco et al., 2010).

\section{Variáveis de personalidade da excelência}

O desempenho superior, em qualquer domínio de atuação, está associado a traços de personalidade facilitadores da emergência das capacidades e potencialidades requeridas para aquela prática. Para além de algumas dimensões da inteligência emocional, como a gestão do estresse ou controle emocional, alguns autores falam no ajustamento ocorrido entre motivações e estilos de personalidade ou, ainda, de alguns traços de personalidade na explicação da excelência (Garcia-Santos et al., 2012; Matos et al., 2011; Runco et al., 2010).

À luz da teoria dos Big Five Fators (McCrae \& Costa Jr., 1997), dimensões como a abertura à experiência, o controle emocional, a sociabilidade e a conscienciosidade são apontadas como fundamentais para a excelência, pelo menos em algumas das áreas de realização (Clayton \& Birren,1980; Ruiz, Sánchez, Durán, \& Jiménez, 2006; Sainz et al., 2011). Várias características de personalidade, de algum modo ligadas à criatividade, aparecem relacionadas com a excelência, como por exemplo a autonomia, a independência e o inconformismo (Runco, 2007). Essas características ajudam o indivíduo a superar padrões e a desejar ir além do estabelecido, podendo também aparecer associadas a problemas emocionais, ainda que estes ocorram em qualquer pessoa independentemente das suas altas habilidades (Fleith, 2007). Na verdade, se existem relatos de pintores eminentes que tiveram problemas mentais, tais como Van Gogh, existiram ainda outros, como por exemplo Matisse e Picasso, ou ainda eminentes cientistas, como Thomas Edison, que não apresentavam distúrbios patológicos de 
personalidade. Estes últimos casos ilustram que o alto potencial criativo e cognitivo não se encontra necessariamente relacionado com a doença mental, traduzindo antes formas particulares ou estilos de funcionamento cognitivo (Wechsler et al., 2015).

O interesse pelo conceito de estilo deve-se ao fato de que permite funcionar como uma ponte entre o pensamento e a personalidade, marcando a individualidade de profissionais excelentes. Kirton (1994), por exemplo, discorre sobre a importância dos estilos inovador e adaptador para se entender o comportamento criativo. Sternberg e Grigorenko (1997) apresentam uma teoria sobre estilos definindo funções (legislativa, executiva e de julgamento), formas (monárquica, oligárquica e anárquica) e abrangências (interna e externa). Por sua vez, Wechsler (2006) demonstrou que cinco diferentes tipos de estilos de pensar (Cauteloso-Reflexivo, Inconformista-Inovador, Lógico-Objetivo, Emocional-Intuitivo, Relacional-Divergente) estavam relacionados com a produção reconhecida ou premiada no Brasil, sendo os mesmos importantes para o reconhecimento da liderança e excelência em diferentes áreas (Mundim \& Wechsler, 2007; Wechsler, Vendramini, \& Oakland, 2012).

\section{Considerações Finais}

Aceitando que a família, a escola e a cultura exercem uma influência central na expressão da excelência, valorizando algumas habilidades em detrimento de outras (Solow, 2001; Virgolim \& Konkiewitz, 2014) ou incentivando, inclusive, mais os homens que as mulheres em determinadas áreas, como as matemáticas e as ciências (Halpern, 2012; Nosek et al., 2009), este artigo centrou-se na proposta de que a excelência profissional requer convergência de variáveis cognitivas, motivacionais e de personalidade. Assim, procurou-se, com esta proposta, demonstrar que a capacidade intelectual, apesar de necessária, não é suficiente para explicar a excelência na idade adulta.

Ao explicitar o que são pessoas com inteligência exitosa, Sternberg (2003) explicita que estas não se limitam a possuir altas capacidades, porém refletem antes sobre quando e onde devem utilizálas de maneira efetiva, integrando outras dimensões da sua personalidade nessa análise e na tomada de decisão. Um amplo leque de variáveis psicológicas determinantes da emergência e manutenção da excelência é atribuído a esses indivíduos, integrando a cognição, a motivação e a personalidade. Segundo Ackerman (1996; 2000), a excelência pode ser entendida como um processo desenvolvimental que se inicia com capacidades superiores as quais delimitam até onde o sujeito pode ir, tendo esse processo uma certa direção para formas concretas de atuação e realização em função das características de personalidade e da motivação.

Em uma breve síntese da investigação na área, pautada sobretudo em medidas de autorrelato e dados autobiográficos, destaca-se, neste artigo, que a excelência profissional requer a convergência de variáveis psicológicas relacionadas com a cognição, a motivação e a personalidade. No que diz respeito à cognição, merece destaque os níveis elevados de habilidade e de conhecimento para se atingir a excelência numa determinada área do exercício profissional. Integra-se aqui um conhecimento estratégico, autorregulado e metacognitivo do indivíduo. Quanto à motivação, ganham particular destaque todas as suas formas intrínseca, bem como a paixão harmoniosa com a profissão, traduzidas no comprometimento do indivíduo com um desempenho de qualidade muito superior. Por último, e relacionando criatividade e personalidade, a excelência profissional está associada a características pessoais, como a abertura à experiência, o inconformismo, a autonomia e a procura de novas ideias relevantes nas produções inovadoras.

Reconhecida a importância dessas características pessoais, estudos futuros poderão voltar-se para as maneiras de se estimular a excelência por meio de mentores, os quais poderão ser representados por familiares, professores, pessoas da comunidade ou do local de trabalho. Considerando que a sociedade necessita de pessoas com nível de excelência para realizar determinadas atividades ou para assumir cargos de liderança em diferentes áreas, deve ser reconhecida a importância de mais pesquisas direcionadas a este tema. 


\section{Referências}

Ackerman, P. L. (1996). A theory of adult intellectual development: Process, personality, interests, and knowledge. Intelligence, 22(2), 229-259.

Ackerman, P. L. (2000). Domain-specific knowledge as the "dark matter" of adult intelligence: $\mathrm{Gf} / \mathrm{Gc}$, personality, and interest correlates. Journals of Gerontology: Psychological Sciences, 55B, 69-84.

Alencar, E. M. L. S. (2007). Características socioemocionais dos superdotados: questões atuais. Psicologia em Estudo, 12(2), 371-378.

Almeida, L. S., Guisande, M. A., \& Ferreira, A. I. (2009). Inteligência: perspectivas teóricas. Coimbra: Livraria Almedina.

Amabile, T. M., \& Mueller, J. S. (2008). Studying creativity, its processes, and its antecedents: An exploration of the componential theory of creativity. In J. Zhou \& C. E. Shalley (Eds.), Handbook of organizational creativity (pp.33-64). New York: Lawrence Erlbaum.

Araújo, L. S., Cruz, J. F. A., \& Almeida, L. S. (2011). A entrevista no estudo da excelência: uma proposta. Psychologica, 52(1), 253-280.

Chase, W. G., \& Simon, H. A. (1973). Perception in chess. Cognitive Psychology, 4, 55-81.

Clayton, V. P., \& Birren, J. E. (1980). The development of wisdom across the life-span: A reexamination of an ancient topic. In P. B. Baltes \& O. G. Brim Jr. (Eds.), Life-span developmentand behavior (Vol.3, pp.103-135). New York: Academic Press.

Csikszentmihalyi, M. (1997). Finding flow. New York: Harper \& Row.

Deci, E. L., \& Ryan, R. M. (2000). The "what" and the "why" of goals pursuits: Human needs and the selfdetermination of behavior. Psychology Inquiry, 11(4), 227-268.

Ericsson, K. A. (2005). Recent advances in expertise research: A commentary on the contributions to the special issue. Applied Cognitive Psychology, 19(2), 233-241.

Ericsson, K. A., \& Lehmann, A. C. (1996). Expert and exceptional performance: Evidence of maximal adaptation to task constraints. Annual Review of Psychology, 47, 273-305.

Ericsson, K. A., Krampe, R.T., \& Tesch-Homer, C. (1993). The role of deliberate practice in the acquisition of expert performance. Psycological Rewiew, 100(3), 363-406.

Fleith, D. S. (2007). Altas habilidades e desenvolvimento socioemocional. In D. S. Fleith \& E. M. L. S. Alencar (Eds.), Desenvolvimento de talentos e altas habilidades (pp.41-50). Porto Alegre: Artes Médicas.

Frost, R. O., Marten, P., Lahart, C., \& Rosenblate, R. (1990). The dimensions of perfectionism. Cognitive Therapy and Research, 14(5), 449-468.
Gable, S. L., \& Haidt, J. (2005). What (and why) is positive psychology? Review of General Psychology, 9(2), 103-110.

Gagné, F. (2004). Transforming gifts into talents: The DMGT as a developmental theory. High Ability Studies, 15(2), 119-147.

Gagné, F. (2015). De los genes al talent: La perspectiva DMGT/CMTD. Revista de Educación, 368, 12-39.

Garcia-Santos, S., \& Almeida, L. S. (2008). Historiometria: um método na investigação da excelência. Sobredotação, 9, 77-86.

Garcia-Santos, S. C., Almeida, L. S., \& Werlang, B. S. G. (2012). Human excellence: The contribution of personality. Paideia, 22(52), 271-279.

Getzels, J. W., \& Jackson, P. J. (1962). Creativity and intelligence: Explorations with gifted students. New York: John Wiley.

Halpern, D. F. (2012). Sex differences in cognitive abilities. New York: Psychology Press.

Jauk, E., Benedek, M., Dunst, B., \& Neubauer, A. C. (2013). Relationship between intelligence and creativity: New support for the threshold hypothesis by means of empirical breakpoint detection. Intelligence, 41(4), 212-221.

Kirton, M. (1994). Adaptors and innovators styles of creativity and problem solving. London: Routledge.

Linley, P. A., Joseph, S., Harrington, S., \& Wood, A. M. (2006). Positive psychology: Past, present, and (possible) future. The Journal of Positive Psychology, 1(1), 3-16.

Lubart, T. (2007). Psicologia da criatividade. Porto Alegre: ArtMed.

Lubinski, D., \& Benbow, C. P. (2000). States of excellence. American Psychologist, 55(1), 137-150.

Lubinski, D., Benbow, C. P., Webb, R. M., \& Bleske-Rechek, A. (2006). Tracking exceptional human capital over two decades. Psychological Science, 17(3), 194-199.

Lubinski, D., Benbow, C. P., Shea, D. L., Eftekhari-Sanjaui, H., \& Halvorson, M. B. J. (2001). Men and women at promise for scientific excellence: Similarity not dissimilarity. Psychological Science, 12(4), 309-317.

Maslow, A. H. (1968). Toward a psychology of being. New York: Van Nostrand.

Matos, D. S., Cruz, J. F. A., \& Almeida, L. S. (2011). Excelência no desporto: para uma compreensão da "arquitectura" psicológica dos atletas de elite. Motricidade, 7(4), 27-41.

McCrae, R. R., \& Costa Jr., P. T. (1997). Personality trait structure as a human universal. American Psychologist, 52(5), 509-516.

McNulty, J. K., \& Fincham, F. D. (2012). Beyond positive psychology? Toward a contextual view of psychological processes and well-being. American Psychologist, 67(2), 101-110. 
Monteiro, S. C., Almeida, L. S., \& Vasconcelos, R. M. (2012). Abordagens à aprendizagem, autorregulação e motivação: convergência no desempenho acadêmico excelente. Revista Brasileira de Orientação Profissional, 13(2), 153-162.

Monteiro, S., Castro, M., Almeida, L., \& Cruz, J. F. A. (2009). Alunos de excelência no ensino superior: comunalidades e singularidades na trajectória académica. Análise Psicológica, 1(XVII), 79-87.

Mundim, M. C., \& Wechsler, S. M. (2007). Estilos de pensar e criar em gerentes organizacionais e subordinados. Boletim de Psicologia, 126 (XVII), 15-32.

Nakano, T. C. (2007). Criatividade: características da produção científica brasileira. Avaliação Psicológica, 6(2), 261-270.

Nosek, B. A., Smyth, F. L., Sriram, N., Lindner, N. M., Devos, T., Ayala, A., ... Greenwald, A. G. (2009). National differences in gender-science stereotypes predict national sex-differences in Science and Math achievement. Proceedings of the National Academy of Science, 106(26), 10593-10597.

Nusbaum, E. C., \& Silvia, P. J. (2011). Are intelligence and creativity really so different? Fluid intelligence, executive processes, and strategy use in divergent thinking. Intelligence, 39(1), 36-45.

Pfeiffer, S., \& Wechsler, S. M. (2013). Youth leadership: A proposal for identifying and developing creativity and giftedness. Estudos de Psicologia (Campinas), 30(2), 219-229. http://dx.doi.org/10.1590/S0103-16 6×2013000200008

Primi, R. (2002). Complexity of geometric inductive reasoning tasks: Contribution to the understanding of fluid intelligence. Intelligence, 30(1), 41-70.

Renzulli, J. S. (1986). The three-ring conception of giftedness: A developmental model for creative productivity. In R. Sternberg \& J. E. Davidson (Eds.), Conceptions of giftedness (pp.53-92). Cambridge: Cambridge University Press.

Renzulli, J. S. (2002). Emerging conceptions of giftedness: Building a bridge to the new century. Exceptionality, 10(2), 67-75.

Renzulli, J. S., \& Gaesser, A. H. (2015). Un sistema multicriterial para la identificación del alumnado de alto rendiminento y de alta capacidad creativo-productiva. Revista de Educación, 368, 96-131.

Rogers, C. R. (1961). On becoming a person. Boston: Houghton Mifflin Company.

Ruiz, L. M., Sánchez, M., Durán, J., \& Jiménez, C. (2006). Los expertos en el deporte: su estudio y análisis desde una perspectiva psicológica. Anales de Psicología, 22(1), 132-142.

Runco, M. (2007). Creativity. Theories and themes: Research, development and practice. Burlington, MA:

774 Elsevier Academic Press.
Runco, M., Millar, G., Acar, S., \& Cramond, B. (2010). Torrance tests of creative thinking as predictors of personal and public achievement: A fifty-year follow-up. Creativity Research Journal, 22(4), 361-368.

Ryan, R. M., \& Deci, E. L. (2004). Autonomy is no illusion: Self-determination theory and the empirical study of authenticity, awareness, and will. In J. Greenberg, S. L. Koole, \& T. Pyszczynski (Eds.), Handbook of experimental existential psychology (pp.449-479). New York: The Guilford Press.

Sainz, M., Soto, G., Almeida, L., Ferrándiz, C., Fernández, M. C., \& Ferrando, M. (2011). Componentes socioemocionales y creatividad según nivel de inteligencia. Revista Electrónica de Formación del Profesorado, 14(3), 97-106.

Seligman, M. (2002). Positive psychology, positive prevention, and positive therapy. In C. R. Snyder \& S. J. Lopez (Eds.), Handbook of positive psychology (pp.3-9). New York: Oxford University Press.

Seligman, M., \& Csikszentmihalyi, M. (2000). Positive Psychology: An introduction. American Psychologist, 55(1), 5-14.

Shanteau, J., Weiss, D. J., Thomas, R. P., \& Pounds, J. C. (2002). Performance-based assessment of expertise: How to decide if someone is an expert or not. European Journal of Operational Research, 136(2), 253-263.

Sheldon, K. M., \& King, L. (2001). Why positive psychology is necessary. American Psychologist, 56(3), 216-217.

Solow, R. (2001). Parents' conceptions of giftedness. Gifted Child Today, 24(2), 14-22.

Sternberg, R. J. (1998). Metacognition, abilities, and developing expertise: What makes an expert student? Instructional Science, 26, 127-140.

Sternberg, R. J. (2001). Giftedness as developing expertise: A theory of the interface between high abilities and achieved excellence. High Ability Studies, 12(2), 159-179.

Sternberg, R. J. (2003). A broad view of intelligence: The theory of successful intelligence. Consulting Psychology Journal: Practice and Research, 55(3), 139-154.

Sternberg, R. J., \& Grigorenko, E. (1997). Are cognitive styles still in style? American Psychologist, 52(7), 700-712.

Stoeber, J., \& Otto, K. (2006). Positive perfectionism: Conceptions, evidence, challenges. Personality and Social Psychology Review, 10, 219-319.

Trost, G. (2000). Prediction of excellence in school, higher education and work. In K. Heller, F. Mönks, R. Sternberg, \& R. Subotnik (Eds.), International handbook of giftedness and talent ( $2^{\text {nd }}$ ed.). Oxford: Pergamon Press.

Vallerand, R. J. (2008). On the psychology of passion: In search of what makes people's lives most worth living. Canadian Psychology, 49(1), 1-13. 
Vallerand, R. J. (2010). On passion for life activities: The dualistic model of passion. In M. P. Zanna (Ed.), Advances in experimental social psychology (Vol.42, pp.97-193). New York: Academic Press.

Virgolim, A. M. R., \& Konkiewitz, E. C. (2014). Altas habilidades/superdotação, inteligência e criatividade. Campinas: Papirus Editora.

Wechsler, S. M. (2006). Estilos de pensar e criar. Campinas: LAMP/IDB digital.

Wechsler, S. M. (2008). Criatividade: descobrindo e encorajando ( $3^{a}$ ed.). Campinas: LAMP/PUC-Campinas.

Wechsler, S. M., Oliveira, K. L., \& Suárez, J. T. (2015). Criatividade e saúde mental: desenvolvendo as forças positivas do caráter. In M. F. Morais, L. C. Miranda, \& S. M. Wechsler (Eds.), Criatividade: aplicações práticas em contextos internacionais (pp.59-76). São Paulo: Vetor.

Wechsler, S. M., Vendramini, C., \& Oakland, T. (2012). Thinking and creative styles: A validity study. Creativity Research Journal, 24(2-3), 235-242.

Winner, E. (2000). The origins and ends of giftedness. American Psychologist, 55(1), 159-169.

Zimmerman, B. J. (2002). Achieving academic excellence: A self-regulatory perspective. In M. Ferrari (Ed.), The pursuit of excellence in education (pp.85-110). Hillsdale, NJ: Erlbaum.

Recebido: fevereiro 6, 2015

Versão final: junho 19, 2015

Aprovado: junho 20, 2015 
